\title{
UNA “PREMESSA METODOLOGICA” PER IL PASSAGENWERK: UNA GENEALOGIA DEL CONCETTO BENJAMINIANO DI STORIA
}

Graziano Mazzocchini

\begin{abstract}
RESUMO
O presente artigo propõe-se a reconstrução da gênese de algumas das principais noções que figuram nas Teses Sobre o conceito de história de Walter Benjamin, tais quais a de mônada, a de imagem dialética, a do agora do conhecimento histórico, através da primeira aparição destas próprias noções na grande obra inacabada à qual dedicou-se o filosofo judeu alemão na última década de sua existência, o trabalho sobre as Passagens (Passagenwerk). Buscamos assim propor uma entre várias genealogias possíveis das próprias Teses de 1940, entendendo que com isto a teoria do conhecimento e da práxis do sujeito e do objeto históricos, tal como foi formulada naquele texto, poderá resultar mais inteligível.
\end{abstract}

Palavras-chaves: Passagenwerk. Teses de 1940. Imagem dialética. Fetiche. Imagens de sonho. Mônada. Catástrofe

\section{A "METHODOLOGICAL PREMISE" BY PASSAGENWERK: A GENEALOGY OF THE BENJAMINIAN CONCEPT OF HISTORY}

\begin{abstract}
The present article proposes the reconstruction of the genesis of some of the main notions that appear in the Theses On the concept of history of Walter Benjamin, such as the one of monad, the one of dialectical image, the one of the now of the historical knowledge, through the first apparition of these very notions in the great unfinished work to which the German Jewish philosopher dedicated himself in the last decade of his existence, the Passagewerk. We have thus sought to propose one of several possible genealogies of the 1940 Theses themselves, understanding that in this way the theory of knowledge and praxis of the historical subject and historical object, as formulated in that text, may be more intelligible.
\end{abstract}

Key-words: Passagenwerk. 1940 Theses. Dialectical image. Fetish. Dream image. Monad. Catastrophe 
A partire dal 1927 Walter Benjamin comincia ad attendere ad un'opera che lo terrá in realtá impegnato per tutto il corso successivo della sua esistenza: si tratta del lavoro sui Passages parigini, il cosiddetto Passagenwerk ( 0 Passagenarbeit), il quale rimarrá in veritá incompiuto. Esso non ci è stato restituito se non nella forma di una mole sterminata di appunti, annotazioni e citazioni, e di due Exposés, rispettivamente del 1935 e del 1939, recanti il medesimo titolo: Parigi, capitale del $X I X^{\circ}$ secolo. Sulla scorta delle stesse Lettere di Benjamin, apprendiamo che mentre nel corso della stesura dei primi appunti (1927-1928), quest'opera gli si prospettava come un lavoro $<$ di poche settimane>> (BENJAMIN 2000, 1029), giá nel marzo 1928 egli ammetteva che essa $<<$ [avrebbe potuto] assumere dimensioni molto maggiori...di quelle previste $>>$ ( BENJAMIN 2000, 1031). La storia redazionale del Passagenwerk fu in effetti travagliata, come attestano i ripensamenti di Benjamin in corso d'opera circa l'idea della sua esposizione e le critiche rivoltegli da Adorno a piú riprese (Cfr.BENJAMIN 2000, 1022-1175). Quale materia aveva per oggetto il lavoro sui Passages di Parigi? Dai Primi Appunti

\begin{abstract}
possiamo trarre una sorta di catalogo di temi che permette di capire di cosa, in questo stadio, si dovesse trattare: si parla di strade e grandi magazzini, di panorami, esposizioni mondiali e tipi di illuminazione, di moda, pubblicita e prostituzione, del collezionista, del flaneur e del giocatore, della noia. I passages sono qui solo un tema tra i tanti. Essi fanno parte di quei fenomeni urbanistici comparsi agli albori del secolo xix con l'enfatica pretesa di essere il nuovo ma che erano ormai privi di funzione. ( TIEDEMANN apud BENJAMIN 2000, XIII)
\end{abstract}

L'esposizione diretta di tali fenomeni, senza il corollario di un'interpretazione trascendentale-categoriale, assolveva ad un principio metodologico che giá Benjamin aveva messo a punto nella raccolta aforistica Strada a senso unico (1928): un principio per cosí dire "micrologico", inteso cioè a selezionare e sviscerare le myricae della realtá, i piccoli dettagli marginali che tuttavia possono restituire, alla stregua di cristalli, una totalitá che in essi si racchiude:

Recente invenzione del lusso industriale, questi passages sono corridoi ricoperti di vetro e dalle pareti rivestite di marmo, che attraversano interi caseggiati, i cui proprietari si sono uniti per queste speculazioni. Sui due lati di questi corridoi, che ricevono luce dall'alto, si succedono i più eleganti negozi, sicchè un passage del genere è una citta, un mondo in miniatura ( BENJAMIN 2000, 41) 
Figure rivelaorie della costituzione del moderno sono appunto per Benjamin i Passages, cioè i corridoi che davano ai loro lati su vetrine commerciali, come pure i panorami, le esposizioni universali, i boulevards parigini. Piú specificamente poi gli stessi Passages esprimono la coscienza immediata del moderno, ossia la tendenza di ciascuna epoca a cogliersi come soglia transitoria, luogo di passaggio- appunto- tra una pre-istoria ed una post-storia ${ }^{1}$ :

\begin{abstract}
Non c'é mai stata un'epoca che non si sia sentita...moderna e non abbia creduto di trovarsi immediatamente davanti ad un abisso. La consapevolezza disperatamente lucida di stare nel mezzo di una crisi decisiva è qualcosa di cronico nell'umanitá. Ogni epoca si presenta irrimediabilmente moderna. II "moderno" tuttavia è diverso nel senso in cui sono diverse le varie figure di uno stesso caleidoscopio. ( BENJAMIN 2000, 610)
\end{abstract}

Quale sia la fondamentale critica benjaminiana della modernitá (qui accennata alla fine dell'appunto) cosí intesa diremo piú avanti, per ora approfondiamo i temi filosofico-storici rinvenibili nei primi schizzi del Passagenwerk. Quest'ultimo si presentava dunque inizialmente come <<una fantasmagoria dialettica > ( TIEDEMANN in BENJAMIN 2000, 1026) in quanto si trattava precisamente per Benjamin di esercitare la filosofia direttamente su singolari manifestazioni concrete <<senza preoccuparsi del macchinario sferragliante della filosofia scolastica con le sue tavole trascendentali di comandamenti e di divieti>> ( TIEDEMANN in BENJAMIN 2000, XIII). Qui occorre peró fare subito una precisazione di ordine metodologico: sebbene Benjamin ricusasse una fondazione di tipo trascendentale per l'esposizione del suo oggetto d'indagine, tuttavia nei Passagen non siamo posti ipso facto dinanzi ad un puro e semplice accumulo di citazioni e di materiali "grezzi". Per Benjamin si trattava certamente di "far parlare" gli stessi materiali che adduceva, ma pur sempre nei termini del montaggio e del commentario ad una realtá:

\footnotetext{
${ }^{1}$ Cfr. a tale proposito il seguente appunto a proposito della categoria dell'attualizzazione in cui si configurano gli autentici (in quanto realmente nuovi) fenomeni storici:<< La pre- e post-storia di un fatto storico appaiono in esso grazie alla sua rappresentazione dialettica. E ancora: ogni fatto storico rappresentato dialetticamente si polarizza e diventa un campo di forze in cui si svolge il confronto tra la sua pre- e post-storia. Si trasforma in questo modo, poiche l'attualità agisce dentro di esso.Per questo il fatto storico si polarizza secondo la sua pre e poststoria sempre di nuovo e mai allo stesso modo. E lo fa al di fuori di sè, nell'attualita stessa; come una linea, divisa dal taglio apollineo, percepisce la sua divisione al di la di se stessa.>> (BENJAMIN 2000, 527)

Mestre em Filosofia pela Universitá degli Studi di Bologna (Itália) e Doutorando em Filosofia Contemporânea pela UFMG. Italiano, residente em Belo Horizonte - MG. E-mail:
} 
Metodo di questo lavoro: montaggio letterario. Non ho nulla da dire. Solo da mostrare. Non sottrarrò nulla di prezioso e non mi approprierò di alcuna espressione ingegnosa. Stracci e rifiuti, invece, ma non per farne l'inventario, bensi per rendere loro giustizia nell'unico modo possibile: usandoli.

[...]

Tenere sempre presente che il commento a una realta (poiché qui si tratta del commento, di una interpretazione nei particolari) richiede tutt'altro metodo di quello a un testo. In un caso la scienza fondamentale è la teologia, nell'altro la filologia. (BENJAMIN 2000, 514)

Certo bisogna ancora precisare qui che le citazioni appartengono al Konvolunt $N$, quindi ad un gruppo di appunto piú tardo rispetto ai progetti della prima stesura; tuttavia queste dichiarazioni bastano da sole a restituirci la metodologia benjaminiana dell'esposizione: come nella Premessa gnoseologica al Dramma barocco tedesco (1926) egli intendeva delucidare l'idea inscritta nel fenomeno attraverso l' indicazione, la mostra diretta del fenomeno, cosí qui il concetto del moderno è dispiegato mediante l'esposizione dei dettagli micrologici dei suoi oggetti ( il "commentario alla realtá"). In altri termini, l'idea della modernitá non è giá sottesa ai suoi fenomeni, bensí proprio l'esposizione di questi ultimi, il montaggio dei loro aspetti, ne consente la costruzione. Chiusa questa parentesi metodologica, torniamo ora al contenuto del lavoro sui Passages. Quest'ultimo, si è detto, si proponeva come fantasmagoria, ma anche piú specificamente, come una fantasmagoria dialettica: gli oggetti presi in considerazione contenevano giá in sé, per Benjamin, oltre che la loro stessa esistenza immediata anche un riposto indice segreto che costituisce un rimando all'epoca presente. Ma come svelare questo indice intrinseco? $\mathrm{O}$, in altri termini, come cogliere nelle vestigia del secolo XIX una costitutiva eccedenza che trasponga questi medesimi oggetti di un passato prossimo nel presente stesso? Nei primi materiali dei Passages Benjamin chiarisce questo punto designando gli strati della realtá esaminati come strati onirici, realtá di sogno. Sotto questo rispetto essi sono appunto immagine onirica, analoghe cioè alle sintesi degli stati del sogno che si compiono al momento del risveglio. In quanto immagini oniriche, ciò che è contenuto in esse, rimanda allo stato della veglia, ossia è chiarito nel momento del risveglio, quando se ne acquista consapevolezza. Sulla scorta di tale analogia, il passato prossimo e l'istante presente della sua conoscibilitá equivalgono rispettivamente al sogno e al risveglio dal sogno: il primo diviene intelligibile nel secondo in virtú della 
sintesi che se ne ha nell'appercezione. Pertanto Benjamin segna qui una vera e propria "rivoluzione copernicana" della conoscenza storica: il passato non costituisce piú un <<punto fisso >> a cui il presente debba sforzare di accostarsi, bensí si definisce dialetticamente nell'istante presente in cui balena alla conoscibilitá. Tale rivolgimento radicale del sogno nella veglia, del passato nel presente viene bene illustrato da Benjamin nei seguenti appunti per i Passages:

\begin{abstract}
La svolta copernicana nella visione storica e la seguente: si considerava il «passato» come un punto fisso e si assegnava al presente lo sforzo di far avvicinare a tentoni la conoscenza a questo punto fermo. Ora questo rapporto deve capovolgersi e il passato deve ottenere la sua fissazione dialettica dalla sintesi che il risveglio compie con le sue antitetiche immagini di sogno. La politica consegue il primato sulla storia, cioe $\mathrm{i}$ 《<atti \ storici diventano qualcosa che ci e accaduto giusto in quell'istante; fissarli e compito del ricordo. E il risveglio rappresenta il caso esemplare del ricordare: quel caso in cui riusciamo a ricordarci di cio che e piu prossimo, piu a portata di mano (dell'io)...C'è un sapere "non ancora cosciente" di ciò che è stato, la cui estrazione alla superficie ha la struttura del risveglio.

[...]

Struttura dialettica del risveglio: ricordo e risveglio sono strettamente affini. II risveglio è cioè la svolta copernicana e dialettica della rammemorazione. È un capovolgimento dialettico straordinariamente composito dal mondo del sognatore al mondo dei desti.[...] II nuovo metodo della scienza storica insegna ad esperire il passato nello spirito con la rapiditá e l'intensitá del sogno, per esperire il presente come mondo della veglia, a cui in ultima analisi ogni sogno si riferisce. (BENJAMIN 2000, 971-972)
\end{abstract}

Questi due estratti da soli racchiudono già diversi punti importanti per la comprensione delle Tesi Sul concetto di storia del 1940. In primo luogo, è qui delucidato il profilo gnoseologico della rammemorazione (Eingedenken ), in riferimento alla $<<$ struttura del risveglio $>>$. Come il risveglio fissa il contenuto del sogno in un'immagine onirica, ossia in una sintesi di elementi frammentari, cosí la rammemorazione non coincide giá con la fissazione del passato quale esso effettivamente è stato, bensí secondo un'immagine balenante, pronta a dileguare: l'esperienza del passato si effettua qui <<con la rapiditá e l'intensitá del sogno>>. La corrispondenza strutturale di tale configurazione del ricordo e, correlatamente, di quella dell'autentico oggetto storico con lo statuto che é accordato a questa nel testo delle Tesi è rinvenibile con estrema nitidezza nel seguente brano della Tesi VI:

Mestre em Filosofia pela Universitá degli Studi di Bologna (Itália) e Doutorando em Filosofia Contemporânea pela UFMG. Italiano, residente em Belo Horizonte - MG. E-mail: 


\begin{abstract}
Articolare storicamente il passato non significa conoscerlo $<<$ proprio come è stato davvero >>. Vuole dire impossessarsi di un ricordo cosí come balena in un attimo di pericolo. Per il materialista storico l'importante è trattenere un'immagine del passato nel modo in cui si impone imprevista al soggetto storico nell'attimo del pericolo, che minaccia tanto l'esistenza stessa della tradizione quanto i suoi destinatari. Per entrambi il pericolo è uno solo: prestarsi ad essere strumento della classe dominante. In ogni epoca bisogna tentare di strappare nuovamente la trasmissione del passato al conformismo che è sul punto di soggiogarla...(BENJAMIN 2009, 27)
\end{abstract}

In secondo luogo viene introdotta la nozione cruciale di immagine (Bild), la quale in altri materiali dei Passages è precisata come immagine dialettica. Quest'ultima vale qui semanticamente come vero e proprio antesignano della nozione di monade- quale la si ritrova nelle Tesi. Come infatti attesta un altro appunto piú tardivo, tale immagine dialettica rispecchia in sè la totalitá del $<<$ tempo reale>>, solo peró come miniatura od oggetto micrologico:

Sull'immagine dialettica. In essa si trova il tempo.[...] II tempo reale entra nell'immagine dialettica non a dimensioni naturali - per tacere della dimensione psicologica - ma nella sua forma piu piccola. II momento temporale nell'immagine dialettica è rilevabile interamente solo attraverso il confronto con un altro concetto. Questo concetto è $1 ' \ll$ adesso della conoscibilita». (BENJAMIN 2000, 951)

Cosí analogamente si configura la monade benjaminiana nella Tesi XVII, laddove il filosofo ebreo tedesco qualifica lo scarto tra il proprio concetto di storiografia materialista e ciò che egli definisce storicismo (Historismus)designando con tale definizione quel tipo di storiografia che si limiti all'accumulo di fatti storici grezzi, come tali indifferenti gli uni e agli altri e pertanto perfettamente sostituibili nel <<tempo omogeneo e vuoto>> (Tesi XVII a, BENJAMIN 2009, 55):

Per contro alla base della storiografia materialistica sta un principio costruttivo. Proprio del pensiero non è solo il movimento delle idee, ma anche il loro arresto. Quando il pensiero si arresta d'improvviso in una costellazione satura di tensioni, le provoca un urto in forza del quale essa si cristallizza come monade. II materialista storico si accosta a un oggetto storico solo ed esclusivamente allorquando questo gli si fa incontro come monade.[...] II profitto del...procedere [ del materialista storico] consiste nel fatto che in un'opera è custodita e conservata tutta l'opera, nell'opera intera l'epoca e nell'epoca l'intero corso della storia. II frutto nutriente di ciò che viene compreso storicamente ha al suo interno, come seme prezioso, ma privo di sapore, il tempo. (BENJAMIN 2009, 52-53) 
Infine (ma non da ultimo) è annunciato il primato conseguito dalla politica sulla storia in questa struttura del risveglio: se gli stati coscienziali della veglia compiono una sintesi tra elementi frammentari (discontinui), allora per analogia l'immagine del passato quale è fissata dal ricordo si configura come una combinazione, sulla quale decide prioritariamente l'azione umana od un pensiero strategico. Insomma l'immagine del passato reca con sé il sigillo dell'attualitá: un determinato ricordo affiora alla consapevolezza collettiva esattamente nel momento in cui esso urge al presente, coincide con una possibilitá sulla quale è urgente che si decida, in un senso come in un altro. Giunti a questo punto dell'analisi, proprio l'immagine dialettica puó rischiarare la critica benjaminiana della modernitá contenuta nei Passages. Stando alla definizione del moderno come passage, esso si rappresenta appunto nella coscienza collettiva alla stregua di una soglia prospiciente verso il sempre nuovo: ma questo essenziale carattere di nouveauté che si pensa inerisca al moderno non differisce sostanzialmente, come osserva Benjamin (2000, 6-7; 611), dalla concezione mitico-arcaica dell'accadere. Questa concezione mitica fa sí che l'accadere storico si configuri come <<apparenza>> od eterno ritorno $<<$ del sempreuguale>> (BENJAMIN 2000, 531): la filosofia "zoroastriana" di Nietzsche è appunto una figura emblematica di tale concezione nel XIX secolo (Cfr. BENJAMIN 2000, 125-126; 366). Pertanto questo carattere di novitá costituisce parimenti una pura apparenza, una falsa coscienza del moderno: esso non sta a rappresentare giá la storia originaria della modernitá stessa, bensí postula il riproporsi del sempre eguale nelle vesti del sempre nuovo ( BENJAMIN 2000, 128). La formazione di immagini dialettiche puó quindi strappare all'incantesimo sognante la coscienza collettiva di un'epoca: se lo stesso passage puó divenire immagine dialettica in virtú della sua unitá (sintesi) di strada e dimora ( BENJAMIN 2000, 942), allora in esso puó irrompere una cesura critica nel movimento del pensiero, per cui un passato determinato viene ad incontrarsi con l' <<adesso della sua conoscibilità>> ( BENJAMIN 2000, 951). In tale costellazione tra uno stato passato e l'adesso divengono parimenti definibili e leggibili quello stesso passato e l'epoca presente; in essa cioè entrambi si compenetrano nel pensiero consentendo alla loro reciproca concoscibilitá: in 
questo punto sono spezzate parimenti l'idea mitico-arcaica dell'eterno ritorno intravista come apparenza della storia originaria del moderno $(2000,125)$ e l'idea di un progresso infinito ed indefinito di cui si dimostra l'insostenibilitá, assieme alla filosofia della storia che vi sottende, nelle Tesi (cfr. BENJAMIN 2009, 45). Abbiamo accennato piú sopra al fatto che Benjamin impresse una modificazione ai Passages in corso d'opera, elaborando un altro piano di stesura. Cronologicamente tale torsione va datata tra l'autunno del 1929 e l'inizio del 1934, lasso di tempo durante il quale egli non si dedicó effettivamente all'opera (TIEDEMANN in BENJAMIN 2000, XX-XXI). Fu infatti a seguito di colloqui a Francoforte con Adorno e Horkheimer che egli valutó l'inadeguatezza dei primi piani di stesura : la <<forma romantica>> e l' <<ingenuitá rapsodica>> (BENJAMIN 2000, 1077) di quei primi appunti non potevano assolvere, a rigore, al compito di una storia sociale della Parigi capitale del Secondo Impero. Come attestato in una sua Lettera a Gershom Scholem del 20 gennaio 1930 ( BENJAMIN 2000, 1044), per il prosieguo del lavoro Benjamin si prefiggeva ora l'approfondimento di aspetti tanto della filosofia hegeliana quanto del Capitale di Marx. Proprio nel Libro I del Capitale Benjamin aveva rinvenuto il tema del feticismo delle merci, da lui identificato con un'altra espressione marxiana, quella di fantasmagoria, intesa come la reificazione della coscienza: i rapporti sociali tra gli uomini divengono rapporti tra cose, ossia tanto i rapporti tra i prodotti del lavoro sociale quanto i rapporti tra i produttori si autonomizzano rispetto ai produttori stessi. Proprio in virtú di tale identificazione terminologica il concetto di feticismo in Benjamin non è perfettamente sovrapponibile a quello marxiano. Se per Marx infatti il feticismo della forma-merce è prodotto appunto dall'oggettivazione dei caratteri sociali del lavoro, i quali cosí si estraniano dagli stessi produttori, tuttavia il rapporto sociale tra cose o tra i prodotti del lavoro esprime parimenti i rapporti sociali tra produttori quali essi sono nelle condizioni capitalistiche della produzione (MARX 2009,148-152). Per Benjamin invece il carattere di feticcio della merce implica una forte discrasia tra la societá delle merci in sè e quale si rappresenta- come essa è per sé. In una parola, il feticismo della merce per Benjamin è qualcosa che pertiene piú propriamente all' aspetto esteriore dei prodotti sociali, al loro puro apparire: ne consegue logicamente che, spezzata 
questa pura apparenza o fantasmagoria della merce, sia infranto il mondo stesso delle merci all'interno della coscienza collettiva dei moderni (cfr. BENJAMIN 2000, 987). Qui siamo appunto alla genesi della seconda stesura del lavoro: i motivi strutturali dei passages, del flâneur, dei boulevards e delle strade di Parigi rimanevano invariati, come pure il progetto di un'analisi micrologica delle forme della vita sociale nella metropoli europea; ma ora tale impianto di costruzione poteva vantare fondamenta teoretiche piú solide, derivanti dall'approfondimento della teoria critica marxiana del capitalismo (TIEDEMANN in BENJAMIN 2000, 1049). L'esposizione universale parigina diveniva pertanto ora per Benjamin la stessa auto-rappresentazione fantasmagorica del moderno, luogo di edificazione del mondo delle merci. Nell' Exposé del 1935 viene enunciata in modo cristallino questa stretta correlazione tra passages, immagine di sogno, immagine dialettica e feticismo delle merci:

[...] Ma proprio la modernitá cita sempre la storia originaria. Ciò accade, qui, attraversol'ambiguitá che è propria dei rapporti e dei prodotti sociali dell'epoca. Ambiguitá è l'apparizione figurata della dialettica, la legge della dialettica nell'immobilitá. Questo arresto, o immobilitá, è utopia, e l'immagine dialettica un'immagine di sogno. Un'immagine del genere sono i passages, che sono casa come sono strade. Un'immagine del genere è la prostituta, che è insieme venditrice e merce.(BENJAMIN $2000,14)$

In relazione alla novità teoretica del feticismo delle merci, l'immagine dialettica si configura come costitutiva ambiguitá. Costitutiva ambiguitá nel senso che in tali immagini- come si è accennato- si ritrovano compresi assieme in maniera immanente sia il passato arcaico che una tensione utopica; od in altri termini si ritrovano compresenti i due lati propri del prodotto sociale reificato: il suo valore d'uso oramai occultato, ossia la sua preistoria, ed il suo valore di scambio, ossia la sua apertura ad altri infiniti valori indefinitamente intercambiabili nel futuro. Sotto entrambi i rispetti di questa ambiguitá immanente, la merce e l'esposizione nelle gallerie formano altrettante immagini dialettiche: vale a dire qui immagini del pensiero dialettico in stato di arresto, unitá ancora indecisa dei due poli. II carattere onirico, di incantesimo della coscienza collettiva attribuito a tali immagini sta proprio qui: nell'ambivalenza di arcaico ed assoluta novitá, esse alludono ad una possibile alteritá; ma stando a quanto ebbe modo poi di puntualizzare Benjamin (BENJAMIN apud DESIDERI; BALDI 2010, 154), tale 
possibilitá diviene attuale nel rapporto tra immagine dialettica e risveglio della coscienza. Un rapporto che, in ultima analisi, percorre le medesime figure oniriche delle immagini dialettiche, e che pertanto puó provocare una rottura di queste dall'interno. Proprio questa possibilitá di rottura del feticismo insita nell'immagine dialettica sembrava non avere compreso appieno Theodor Adorno, il quale in una lunga Lettera dell'agosto 1935 ( ADORNO apud BENJAMIN 2000, 1095-1106) rimproverava all' Exposé di avere ridotto il gioco dialettico della coscienza moderna alla dialettica di sogno e veglia, senza avere debitamente svolto il motivo marxiano del feticismo delle merci. Ciò che Adorno rimproverava a Benjamin era, in sintesi, di aver ricondotto integralmente l'immagine dialettica nella sfera della stessa coscienza, sognante e vigile che fosse, invece che considerarla come una trasposizione oggettiva della coscienza collettiva. Inoltre, se Benjamin designava la forma della merce come immagine dialettica, allora tale identificazione avrebbe dovuto comportare coerentemente che la merce come immagine del moderno si estinguesse da un lato nel suo valore d'uso, ma dall'altro divenisse l'immagine propria della promessa d'immortalitá per la coscienza dei moderni:

\begin{abstract}
Intendere la merce come immagine dialettica significa appunto anche intenderla come motivo del suo tramonto e del suo "superamento" invece che della pura regressione al più antico. La merce da un lato è l'estraniato, nel quale il valore d'uso si estingue, ma dall'altro è anche ciò che sopravvive, che, diventato estraneo, regge all'immediatezza. Nelle merci, e non immediatamente per gli uomini, ci è data la promessa dell'immortalità, e il feticcio è [...] per il diciannovesimo secolo un'infedele ultima immagine [...] ( ADORNO apud DESIDERI; BALDI 2010, 153)
\end{abstract}

Da parte sua Benjamin evitó in larga misura di rispondere puntualmente a queste obiezioni di Adorno, ma poté almeno rivendicare alle sue immagini dialettiche un'evidente potenza critica:

[...] l'immagine dialettica non ricalca il sogno - dire questo non è mai stato mia intenzione. Ma per certo mi pare che essa contenga le istanze, il punto in cui irrompe il risveglio, e che anzi crei la propria figura da questi punti come una costellazione dai punti luminosi. Anche qui dunque un arco da tendere, una dialettica da dominare: la dialettica di immagine e risveglio. ( BENJAMIN apud DESIDERI; BALDI 2010, 154) 
In definitiva, in virtú di questa dialettica tra immagine e risveglio la malia del ritorno sempre eguale dell'arcaico in vesti nuove è infranta, per risolversi in un'autentica storia originaria della modernitá, nella quale cioè gli stessi elementi costitutivi del moderno si articolano in costellazioni tra passato e presente assolutamente inedite, uniche ed irriducibili. Che poi la costruzione dell'oggetto storico autentico sia il frutto della dialettica tra immagine e risveglio è chiarito bene anche da questo appunto dei Passages:

Nell'immagine dialettica, cio che è stato in una determinata epoca e sempre, al tempo stesso, il « da-sempre-gia-stato». Ma ogni volta esso si manifesta come tale solo agli occhi di un'epoca ben precisa: ovvero quella in cui l'umanita, stropicciandosi gli occhi, riconosce come tale proprio quest'immagine di sogno. Ė in quest'attimo che lo storico si assume il compito dell'interpretazione del sogno. ( BENJAMIN 2000, 519-520)

Ora che abbiamo puntualizzato i nodi teoretici costitutivi del lavoro sui Passages, ci vogliamo a prendere in esame quei materiali che possano costituire un vero e proprio sfondo conoscitivo per le Tesi del 1940; in particolare si tratta di appunti contenuti nel cosiddetto Konvolunt $N$, recante il doppio titolo di Elementi di teoria della conoscenza-Teoria del progresso. A proposito della prima dicitura, essa contiene un'indicazione assai preziosa: come attestato anche da svariate Lettere alla cerchia degli amici (Horkheimer, Adorno, Scholem), Benjamin andava progressivamente prefiggendosi, tra il 1935 ed il 1940, una chiarificazione gnoseologica al Passagenwerk. Questo progetto di una premessa metodologica il filosofo tedesco se la prefigurava in analogia con la Premessa gnoseologica al saggio sul Trauerspiel tedesco del 1926. Cosí in una Lettera a Scholem del 20 maggio 1935:

Per il resto cedo talvolta alla tentazione di figurarmi delle analogie con il libro sul barocco nella costruzione interna [...] E voglio accennarti almeno questo: anche qui stará al centro il dispiegamento di un concetto tradizionale. Se là era il concetto di Trauerspiel, qui sarebbe quello del carattere di feticcio della merce. Se il libro sul barocco mobilitava una propria teoria della conoscenza, ciò accadrebbe almeno in uguale misura per i Passagen, benché io non sappia ancora prevedere se essa troverá una rappresentazione autonoma, né in che misura riusciró ad elaborarla. ( BENJAMIN 2009, 7) 
Come si evince dalle altre Lettere al riguardo ( cfr. BENJAMIN 2009, 293328 e BENJAMIN 20001053 sgg.), il <<concetto tradizionale>> dispiegato a preludio gnoseologico dei Passagen consisterá piú precisamente nella disamina sulle teorie del progresso e sul concetto di storia. Procedendo all'esame dei passi piú significativi, prendiamo anzitutto in considerazione un ampio stralcio della nota contrassegnata dalla sigla N 3,1:

\begin{abstract}
L'indice storico delle immagini dice, infatti, non solo che esse appartengono a un'epoca determinata, ma soprattutto che esse giungono a leggibilita solo in un'epoca determinata. E precisamente questo giungere a leggibilità è un determinato punto critico del loro intimo movimento. Ogni presente è determinato da quelle immagini che gli sono sincrone: ogni adesso è I 'adesso di una determinata conoscibilita. In questo adesso la verita è carica di tempo fino a frantumarsi. (E questo frantumarsi,e nient'altro, e la morte dell'intentio, che quindi coincide con la nascita dell'autentico tempo storico, il tempo della verita). Non è che il passato getti la sua luce sul presente 0 il presente la sua luce sul passato, ma immagine è cio in cui quel che è stato si unisce fulmineamente con I 'adesso in una costellazione. In altre parole: immagine è la dialettica nell'immobilità. Poichè mentre la relazione del presente con il passato è puramente temporale, quella tra ciò che è stato e I 'adesso è dialettica: non di natura temporale, ma immaginale. Solo le immagini dialettiche sono immagini autenticamente storiche, cioe non arcaiche. L'immagine letta, vale a dire I 'immagine nell'adesso della leggibilità, porta in sommo grado I 'impronta di questo momento critico e pericoloso che sta alla base di ogni lettura. (BENJAMIN 2000, 517-518)
\end{abstract}

Passato, presente, Jetztzeit o adesso, costellazione, immagine dialettica, istante critico: in questo estratto tutti questi concetti già delineati piú sopra sono disposti in modo organico a formare una teoria della conoscenza storica. Compenetrandosi dialetticamente nelle immagini, il passato ed il presente si risolvono dalla loro fissitá ideale rispettivamente in ciò-che-è-stato e nell'adesso della conoscibilitá. In altri termini, un momento passato contiene in sè l'indice della propria storicitá non in quanto passato fissato una volta per tutte, bensí in quanto rinvia ad un istante presente nel quale soltanto perviene alla propria leggibilitá. In questa prospettiva, in cui viene meno un ordine prioritario logico e cronologico tra passato e presente, tanto un passato determinato quanto un presente determinato si definiscono reciprocamente in virtú della loro sincronia. Tale sincronia poi si costituisce puntualmente entro un istante critico, pericoloso, nel quale una determinata virtualitá passata ritorna fulmineamente nel presente, si congiunge ad esso subitaneamente in una costellazione: solo in quell'istante

Mestre em Filosofia pela Universitá degli Studi di Bologna (Itália) e Doutorando em Filosofia Contemporânea pela UFMG. Italiano, residente em Belo Horizonte - MG. E-mail: 
presente determinato una possibilitá riposta nel passato puó ritornare ad essere riconosciuta come possibilitá presente, suscettibile di divenire attuale ancora una volta. L'istant in cui si compie la costellazione è un istante critico perché malfermo: un attimo ancora, e l'immagine del passato che reca con sé alla conoscenza rischia di rifluire irrimediabilmente, ricadere nell'oblio senza che sia piú rintracciabile (BENJAMIN 2009, 27). Benjamin precisa qui che la relazione tra ciò che è stato e l'adesso della sua leggibilitá è una relazione immaginale, non temporale: momento trascorso e adesso possono riconoscersi l'uno nell'altro proprio in virtú di un'immagine, nella quale l'intero movimento della dialettica si trovi momentaneamente bloccato, in battuta d'arresto. Quest'immagine della dialettica in battuta d'arresto coincide appunto con l'immagine dialettica od ancora con la monade: essa costituisce l'oggetto storico in miniatura, in cui la costellazione dei punti focali della storia si sia cristallizzata. Sempre nell'immagine la costellazione dei punti focali della storia, di passato e presente, è cosí satura di tensioni, cosí tesa nell'adesso della conoscibilitá, che pare sul punto di frantumarsi: se nella Jetztzeit ha luogo l'appropriazione di una determinata possibilitá passata, se in esso cioè questa possibilitá sia attualizzata, allora s'inaugura il <<tempo della veritá>>, vale a dire che questa possibilitá è conquistata al suo autentico senso storico. Un oggetto é pertanto autenticamente storico quando sia estratto dal decorso storico e posto in sincronia con l'istante presente, in cui se ne ha conoscenza, secondo l'immagine in cui è pervenuto subitamente ad offrirsi, Tale estrazione di un'opera determinata del movimento dialettico del pensiero, questa sua attualizzaziomne coincide poi con la salvazione ( Rettung) o redenzione ( Erlösung) di un passato ad opera dello storiografo materialista (cfr. BENJAMIN 2000, 533):

\footnotetext{
L'immagine dialettica è un'immagine balenante. Cio che è stato va trattenuto cosi, come un'immagine che balena nell'adesso della conoscibilita. La salvezza, che in questo modo - e solo in questo modo - è compiuta, si lascia compiere solo in ciò che nell'attimo successivo è gia irrimediabilmente perduto.

[...]

II materialista storico deve abbandonare l'elemento epico della storia. Esso fa saltar fuori l'epoca dalla reificata "continuitá della storia". Ma scardina anche l'omogeneitá dell'epoca. Esso vi frammischia l'ecrasite, cioé il presente. (BENJAMIN 2000, 531; 533)
} 
L'immagine posticcia di un continuum storico è pertanto richiesto l'intervento attivo dello storico, il quale posto dinanzi alla monade ne estrae un'epoca in modo forzoso: si potrebbe dire che l'epoca sia divelta dal corso omogeneo della storia. All'estrazione dell'epoca da tale continuo corrisponde in altri appunti del Konvolunt $N$ una categoria che Benjamin definisce propriamente <<politica>>: la <<presenza di spirito>> ( BENJAMIN 2000, 526; 537) dello storico che adoperi il metodo dialettico. Con tale espressione viene designata in questi passaggi esattamente la prontezza, la tempestivitá dello storico ad accostarsi all'immagine del passato nel suo balenare- al fine preciso di salvarla. Se invece tale tempestiva captazione venga a mancare, allora ciascun punto della <<costellazione di pericoli>> ( BENJAMIN 2000, 526) potrá infine corrispondere al riproporsi della catastrofe: $<<\|$ ll concetto di progresso va fondato nell'idea della catastrofe. Che "tutto continui cosí" è la catastrofe. Essa non è ciò che di volta in volta incombe, ma ciò che di volta in volta è dato.>> ( BENJAMIN $2000,531)$. L'immagine dell'ecrasite vista piú sopra è eloquente a questo proposito: il termine designa infatti una sostanza esplosiva il cui potere raddoppia quello della dinamite. Non é questa l'unica metafora dinamitarda che il filosofo tedesco utilizza per illustrare il lavoro del materialista storico, come si evince da una versione manoscritta alternativa al testo definitivo della Tesi XIV Sul concetto di storia: $<$ Dove il passato è carico di questo materiale esplosivo, la ricerca materialista appone la miccia al continuum omogeneo e vuoto della storia. Con questo procedimento si propone di estrarne a forza un'epoca>> ( BENJAMIN 2009,46 , nota b). Con la deflagrazione del continuum storico ad opera del dialettico è definitivamente infranta anche l'immagine mitica dell'accadere storico come riproporsi del sempre uguale:

\footnotetext{
Per lo storico materialista ogni epoca, di cui egli si occupa, è solo un antefatto dell'epoca cui lui stesso appartiene. E proprio per questo per lui non c'è nella storia I 'apparenza della ripetizione, poiché proprio $\mathrm{i}$ momenti del corso della storia che gli stanno piú a cuore, attraverso il loro essere «antefatto», divengono momenti del presente stesso e mutano il loro carattere a seconda che il loro esito sia catastrofico o trionfale. (BENJAMIN 2000, 532)
}

Piuttosto che ritornare in qualitá di identico, il passato diviene conoscibile in un modo inedito ed assolutamente originale in ciascuna costellazione in cui si 
congiunga con l'adesso. Queste stesse costellazioni si configurano perció a propria volta come uniche ed irriducibili, ossia assolutamente singolari. Strettamente congiunto al tema della continuitá del corso storico si trova un altro tema dirimente per Benjamin, quello della continuitá dell'oppressione nella storia, o piú precisamente il tema della tradizione dei vincitori. L'immagine posticcia di un corso omogeneo della storia risulta in ultima analisi fondata sulla continuitá del dominio: questa intuizione Benjamin la svilupperá nel nel testo delle Tesi VII e VIII ( BENJAMIN 2009, 29-33), mentre nel frammento corrispettivo nel Passagenwerk ( BENJAMIN 2000, 402), la correlazione tra costituzione del dominio di una classe sulle altre e fondazione del tempo storico è esattamente invertita: suscettibile ai cambiamenti storici con la stessa agilitá della moda, la borghesia si adatta bene ai risultati sempre nuovi del conflitto sociale, convertendo questi risultati nelle figure di una realtá sostanzialmente pacificata. Vi sono peró sempre nel Konvolunt $N$ altri cenni significativi al riguardo, che sembrano attestare giá questa intuizione fondamentale:

La celebrazione o apologia si propone di occultare i momenti
rivoluzionari della storia. A essa sta a cuore la produzione di una
continuità. Attribuisce importanza solo a quegli elementi dell'opera che
già sono confluiti negli effetti della stessa. Le sfuggono i punti in cui la
tradizione si interrompe, e di conseguenza anche le asprezze e gli
spuntoni che forniscono un appiglio a chi intende superarla.[...] Puo
darsi che la continuità della tradizione sia un'illusione. Ma allora proprio
la permanenza di questa illusione di permanenza fonda in essa la
continuità. ( BENJAMIN 2000,532; 547)

L'apologia è qui implicitamente l'apologia del dominio, l'apologia di una classe dominante. È questa intenzione apologetica del dominio di classe che costituisce l'immagine di una continuitá del senso storico: in essa non possono darsi punti focali critici, che ne infrangano l'omogeneitá, pena il tramandamento degli istanti di rottura nella coscienza collettiva dei posteri. Secondo il testo della Tesi VII Sul concetto di storia ( BENJAMIN 2009, 29-30) il correlato storiografico di tale fabbricazione della continuitá è costituito dal metodo storicista dell' <<immedesimazione>> con un'epoca passata: recidendo il nesso tra un'epoca passata e quella presente, questo metodo finisce con il considerare un'epoca determinata come giá definitivamente conclusa nelle sue possibilitá, giá assolutamente data, finisce cioè con il celebrare l'apologia dei vincitori di turno.

Mestre em Filosofia pela Universitá degli Studi di Bologna (Itália) e Doutorando em Filosofia Contemporânea pela UFMG. Italiano, residente em Belo Horizonte - MG. E-mail: 
Infine, la stessa scoperta benjaminiana della fondazione di un continuum fittizio della storia sulla continuitá del dominio della borghesia la troviamo ancora, espressa secondo la mirabile metafora del caleidoscopio, negli appunti raccolti nel 1939 sotto il titolo di Zentralpark:

\begin{abstract}
Il corso della storia, cosí come si presenta sotto il concetto di catastrofe, non puó in realtá impegnare il pensatore piú che un caleidoscopio in mano a un bambino, nel quale ad ogni rotazione l'intero ben ordinato rovina verso un ordine nuovo[...] I concetti dei dominatori sono stati ogni volta gli specchi grazie ai quali è venuta a costituirsi l'immagine di un "ordine". Deve essere infranto il caleidoscopio. (BENJAMIN 2010, 131144)
\end{abstract}

La catastrofe, ovvero la chance rivoluzionaria mancata, si rivela in ultima analisi come la reale sostanza della continuitá storica. Le Tesi Sul concetto di storia vorranno pertanto essere anche un piccolo vademecum insieme teorico e pratico per il <<soggetto della conoscenza storica $>$ : $<<<$ la classe degli oppressi che lotta $>$ (BENJAMIN 2009, 43).

\title{
RIFERIMENTI
}

BENJAMIN, Walter. Angelus Novus. Saggi e Frammenti. Traduzione di Renato Solmi. Torino: Einaudi, 2010.

. Opere complete vol.IX. I Passages di Parigi. Edizione italiana a cura di Enrico Gianni. Torino: Einaudi, 2000.

Torino: Einaudi, 2009.

Sul concetto di storia. A cura di G.Bonola e M.Ranchetti.

DESIDERI, F., BALDI, M. Benjamin. Roma: Carocci, 2010.

MARX, Karl. II Capitale. Torino: UTET, 2009.

TIEDEMANN, R. Introduzione a Benjamin. 2000. 
Mestre em Filosofia pela Universitá degli Studi di Bologna (Itália) e Doutorando em Filosofia Contemporânea pela UFMG. Italiano, residente em Belo Horizonte - MG. E-mail: 